Diesen in der Tabelle angeführten Merkmalen ist zur Kenntlichkeit des $A$. leeuweni nur noch wenig hinzuzufügen. Das Halsschild ist wie bei obesus Fst. gekörnelt, aber nicht wie bei diesem an den Hinterecken eingezogen, sondern parallelseitig, so breit wie die Decken; letztere sind kaum $2^{1} /{ }_{3}$ mal so lang wie breit $(7: 3 \mathrm{~mm})$ und relativ etwas schmäler wie bei obesus Faust, denn die Angabe, dals bei diesem die Flügeldecken nahezu $21 /{ }_{2}$ mal so lang wie breit sind (Ann. Mus Genova 1894, XXXIV p. 249), beruht auf einem Versehen, ihre Mafse sind 11:5,5 mm. Die Punkte der nach der Spitze zu furchenartigen Streifen sind hinter der Deckenmitte am gröfsten und hier zuweilen mehr als doppelt so lang wie breit. Die Unterseite des Rüssels zeigt in beiden Geschlechtern eine ziemlich deutliche Mittelleiste, die des Kopfes eine Querrunzelung und die des übrigen Körpers eine mälsig dichte schuppenartige Beborstung, d. h. jedem Punkt entspringt ein Büschel strahlenartig auseinandergehender Borsten, die ganz ähnliches Aussehen zeigen wie die fiederartig zerschlitzten Schuppen, die bei den Cleoniden vorkommen. Auch A. obesus hat ähnliche, jedoch längere Borstenhaare, die namentlich am Hinterrande der 3 vorletzten Segmente mehr pinselartig als strahlenbüschelig angeordnet sind. Vorderschienen grob längsstreifig punktiert, verhältnismälsig stark gekrümmt, die proximale Ausbuchtung des Innenrandes kürzer und etwas tiefer als die distale, die Erweiterung daher hinter der Mitte und sehr stumpf.

\title{
Ein neuer hygrophiler Rüsselkäfer aus Argentinien. (Col.)
}

\section{Von Prof. H. Kolbe, Berlin.}

Der vorliegende Fall lehrt uns wieder, wie gleiche absonderliche Lebensweise sich bei den Angehörigen einer in weit voneinander getrennten Kontinenten vertretenen Gattungsgruppe wiederfindet. Die Gruppe der B a g o i n e n, welche zu den Rüsselkäfern (Curculioniden) gehört, enthält einige Gattungen, die nur an Gewässern gefunden werden und an oder in Wasserpflanzen und teilweise sogar unter dem Wasserspiegel leben. Sehr nahe verwandt sind mit ihnen die Errirhinen und Tanysphyrinen, deren Angehörige teilweise dieselbe Lebensweise haben.

- Vor einiger Zeit entdeckte Herr $\mathrm{H}$ a n s $\mathrm{Richter}$ in Buenos Aires einen solchen im Wasser lebenden Rüsselkäfer, den er zur 
weiteren Beobachtung seiner Lebensweise Herrn Prof. Dr. P. F r a n k übergab. Dieser hat im letzten Heft dieser Zeitschrift S. 141 darüber ausführlich berichtet. Den Käfer schickte Herr H. Richter zur Bestimmung in 2 Exemplaren ein; die nähere Untersuchung ergab, dafs er zu den Errirhine n, und zwar zur Untergruppe der $\mathrm{B}$ a g o in en gehört, weil die Tarsen nur schmal und das dritte Tarsenglied nicht oder kaum breiter ist als das zweite, auch unterhalb keine Bürste aufweist. Ich nenne den Käfer

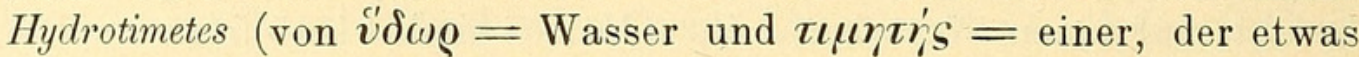
schätzt) natans.

Die Gattung ist im folgenden gekennzeichnet. Der Rüssel ist ungefähr so lang wie der Prothorax, etwas gebogen, länger und dünner als bei der amerikanischen Gattung Lissorhoptrus. Die Antennenkeule ist länglich, dreigliedrig, das letzte Glied tomentiert, die beiden vorletzten Glieder so glänzend wie bei Lissorhoptrus Horn (und Elmidomorphus Europas). Der Funiculus ist sechsgliedrig, erstes Glied dick, zweites Glied dünn, länger als die folgenden, welche an Stärke etwas zunehmen. Das Prosternum ist flach. Die vorderen Coxen sind ziemlich grofs, vorstehend, zusammenstofsend. Die Tibien aller Beine sind mäfsig schlank und an der Spitze mit einem zugespitzten, einwärts gebogenen Haken versehen. An den schmalen Tarsen ist das dritte Glied dünn, nicht breiter als das vorhergehende, das letzte Glied kürzer als die beiden vorhergehenden (bei Lissorhoptrus ebenso lang). Die Tibien sind einfach (nicht gefurcht wie bei Ochetina und Onychylis). Die Tibien des zweiten Beinpaares sind an der Aufsen- und Innenseite lang und reichlich gefranst. Die Tibien des ersten Paares sind nur an der Innenseite, diejenigen des dritten Paares innenseits gegen die Spitze hin gefranst. Die Aufsenseite der Tibien des zweiten Beinpaares ist gebogen, die Innenseite gerade.

Charakteristik der Gattung: Frons aequalis, convexiuscula; oculi ovati, supra et infra inter se separati, paulo convexi, sat grosse granulati. Rostrum sat tenue, curvatum, longitudine prothoracis. Antennae rostro paulo infra medium insertae, mediocres; clava elongata, acuminata, nitida, articulo ultimo opaco; scapus oculum haud attingens; funiculus ad clavam versus sensim incrassatus, articulo 1. crasso ovato, 2. tenui, 3 . et 4 . junctis fere longiore, his et ceteris brevibus, crassioribus. P r othorax latior quam longior, elytris angustior, postice quam antice paulo latior, margine anteriore necnon posteriore obtusatis, lateraliter rotundatus, in dorso convexo, ante marginem anteriorem sulco transverso (medio interrupto) leviter constrictus. Scutell um invisum. Elytra modice elongata, valde convexa, leviter rotundate ampliata, humeris valde obliquatis subobsoletis. 
Metasternum mesosterno duplo longius. Coxa e exsertae, anticae et mediae utraeque inter se conniventes. Fem or a subclavata, recta vel subrecta, mutica, illa secundi paris leviter flexuosa. Tibia e integrae (non sulcatae); illae secundi paris latere exteriore curvatae, intus et extus per totam longitudinem sat longe ciliatae, natatoriae; tibiae primi paris intus paulo ciliatae. T a rsi angusti, elongati, lineares, articulo tertio integro (nec dilatato, nec bilobo); unguiculi liberi. L a mina e a b do$\mathrm{m}$ in is duae basales lateraliter longitudine aequales, lamina secunda laminis 3 . et 4 . junctis paulo longior.

H. natans n. sp. Fusco-griseus, lateraliter et infra flavogriseus; rostro nitido antennisque castaneo-rufis; prothorace quinta parte latiore quam longiore, convexo, supra subtilissime confertim punctulato-coriaceo; elytris striato-foveatis, interstitiis striarum interfovealibus lateraliter et postice tuberculiformibus; tuberculis elytrorum quinque posticis majoribus, tribus in parte dehiscente, ultima singula in apice ipso posito.

Long. corp. (rostro excluso) $3-3^{1 / 4} \mathrm{~mm}$.

Vaterland: Argentinien, Provinz Buenos Aires, von Herrn Hans Richter gesammelt und an das Königl. Zoologische Museum zu Berlin zur Beschreibung eingesandt.

Es gibt noch andere hygrophile Curculioniden. Innerhalb der Fauna Deutschlands ist es zunächst Eubrychius velatus Beck (aquaticus Thoms., der mit Phytobius nahe verwandt ist und zu den Ceutorhynchinen gehört; er lebt in stehendem Wasser, unter dessen Oberfläche an den Stengeln von Wasserpflanzen (Redtenbacher, Fauna Austriaca. II. p. 777). Diese Art ist ein guter Schwimmer, wie bereits der Autor der Spezies, v. B e ck, in seinen „Beiträgen zur bayerischen Insektenkunde" (1817) mitteilt. Auch der nahe verwandte Litodactylus leucogaster Marsh. (myriophylli Gyll.) findet sich im Wasser. Dagegen leben die Arten von Phytobius am Ufer von Flüssen und stehenden Gewässern im Sande. Der auf Wasserpflanzen lebende Lixus paraplecticus steigt zur Eiablage an Pflanzenstengeln in das Wasser hinab. 


\section{$2 \mathrm{BHL}$ Biodiversity Heritage Library}

Kolbe, H. J. 1911. "Ein neuer hygrophiler Rüsselkäfer aus Argentinien. (Col.)." Deutsche entomologische Zeitschrift 1911(3), 315-317. https://doi.org/10.1002/mmnd.48019110307.

View This Item Online: https://www.biodiversitylibrary.org/item/103379

DOI: https://doi.org/10.1002/mmnd.48019110307

Permalink: https://www.biodiversitylibrary.org/partpdf/236056

\section{Holding Institution}

Harvard University, Museum of Comparative Zoology, Ernst Mayr Library

\section{Sponsored by}

Biodiversity Heritage Library

\section{Copyright \& Reuse}

Copyright Status: Public domain. The BHL considers that this work is no longer under copyright protection.

This document was created from content at the Biodiversity Heritage Library, the world's largest open access digital library for biodiversity literature and archives. Visit BHL at https://www.biodiversitylibrary.org. 